\title{
LA SÍTULA DE CAESARAUGUSTA-ZARAGOZA Y LOS APLIQUES TIPO III DE DELGADO
}

\author{
POR \\ ROMANA ERICE LACABE \\ Unidad de Museos. Ayuntamiento de Zaragoza
}

\section{RESUMEN}

Se da a conocer el hallazgo de una sítula completa de Zaragoza que conserva los apliques figurados, unas máscaras masculinas correspondientes al tipo III de Delgado, grupo tipológico poco conocido del que se presenta un primer estudio.

\section{SUMMARY}

This paper deals with a bronze situla from CaesaraugustaZaragoza, which has preserved its handles decorated with male masks type III from Delgado. It is made here the first classification of this group of situlae, until now not well known

PALABRAS CLAVE: Vajilla de bronce, época romana, apliques figurados hispanos.

KEY WORDS: Bronze vessels, Roman time, Hispanic handle attachments decorated.

Este trabajo se propone dar a conocer el singular hallazgo en Zaragoza de una sítula romana completa, que además conserva el asa en su posición original y excepcionalmente los apliques figurados. Estos últimos pertenecen a un grupo tipológico del que poco se conocía hasta la actualidad, como es el tipo III de Delgado. El grupo fue propuesto por M. Delgado en 1970, a través de una tipología en la que daba a conocer cuatro formas de apliques típicamente hispanos aparecidos en las excavaciones de Conímbriga. Ese trabajo supuso la base y punto de partida para el estudio de unas piezas ampliamente difundidas por la Península Ibérica, los tipos I, II y IV de esta autora, mientras que su tipo III estaba constituido por una sola pieza. En este trabajo se muestra una posible relación entre apliques figurados de tipo Delgado y ciertas formas de recipientes. Además, se presenta un catálogo de las formas pertenecientes al tipo III de Delgado, se propone el origen y una posible cronología del mismo y, finalmente, se analiza el objeto y la función de las sítulas.

La sítula apareció en las excavaciones llevadas a cabo en la calle Alcober 10-14 angular a calle Olleta de Zaragoza. El hallazgo tuvo lugar en el interior de un tramo de una pequeña cloaca de cinco metros de longi- tud, situado junto a una domus romana, ubicada intramuros de la ciudad, fechada entre mediados del siglo II y la primera mitad del siglo III d.e ${ }^{1}$.

\section{EL RECIPIENTE (fig. 1)}

La sítula mide $23,5 \mathrm{~cm}$ de alto; $20,4 \mathrm{~cm}$ de diámetro en la boca; $24,5 \mathrm{~cm}$ de diámetro en la carena; $15 \mathrm{~cm}$ de diámetro en el fondo y se encuentra depositada en el Museo de Zaragoza, $\mathrm{n}^{\circ}$ inv. 01.6.1.

La sítula de Caesaraugusta tiene forma bitroncocónica, presenta una carena situada por encima de la mitad de la vasija y su borde es exvasado. El recipiente está elaborado a partir de dos láminas fundidas, trabajadas a martillo hasta darles la forma, unidas en la zona de la carena por una serie de remaches. El borde se ha obtenido por martilleado, volviendo el extremo de la lámina. El fondo, ligeramente cóncavo en el interior, está fabricado aparte, en una sola pieza. Se procedió al martilleado del mismo hasta sobreponer su borde al borde inferior de la vasija, llevando a cabo posteriormente la soldadura de ambas partes. El fondo no presenta huellas de trabajo a torno, mientras que en las paredes interiores de la vasija se observan claramente las líneas de torneado.

La sutura de varias láminas con remaches es una técnica antigua conocida también entre las formas metálicas de tipología romana, así lo muestran los acetres tipo 3a, 3b y 4 de $\mathrm{P}$. $\mathrm{Palol}^{2}$, y tres de las sítulas procedentes Do Fojo das Pombas en Valongo, Portugal ${ }^{3}$.

En la Península Ibérica, las sítulas completas conocidas son escasas, pero las adornadas con apliques figurados incluidas en la tipología de M. Delgado ${ }^{4}$ son casi

\footnotetext{
1 La empresa Arqueoexpert llevó a cabo la excavación en enero de 2001, bajo la dirección de Blanca del Real, a la que agradecemos toda la información ofrecida sobre la descripción del hallazgo de la sítula y sobre el contexto arqueológico de la misma.

2 1970, 233, fig. 11. Se trata además de los tipos 3 y 4 de A. Fuentes, 1990, 124.

3 L. Alcubierre, 1962, fig. 6.8, 6.9, 6.10.

4 1970,15-27.
} 

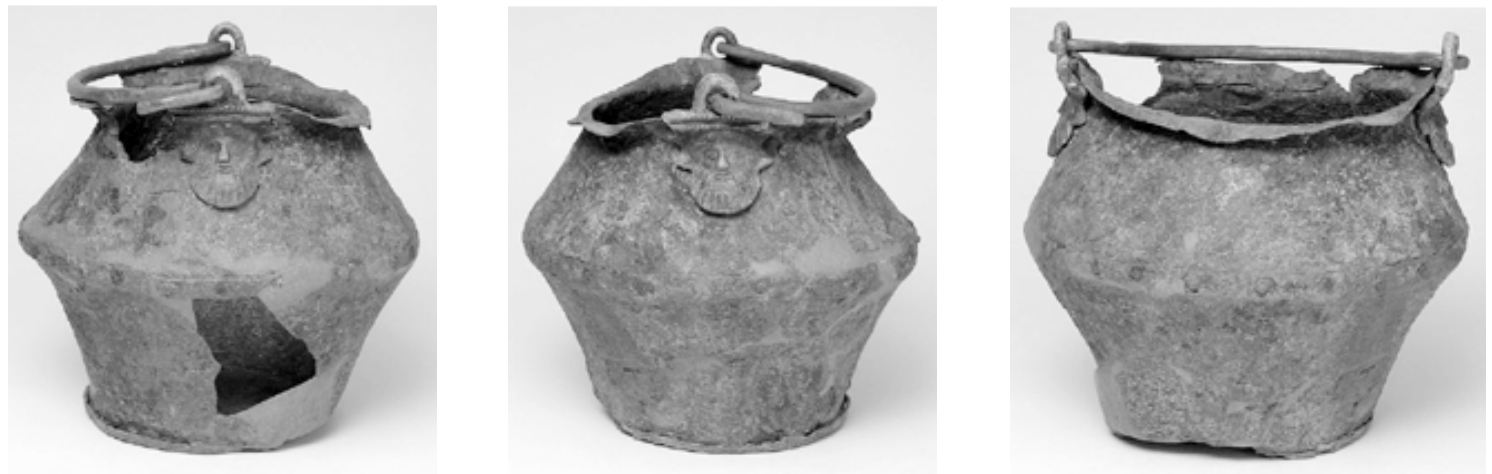

Figura 1. La sítula de Caesarangusta.
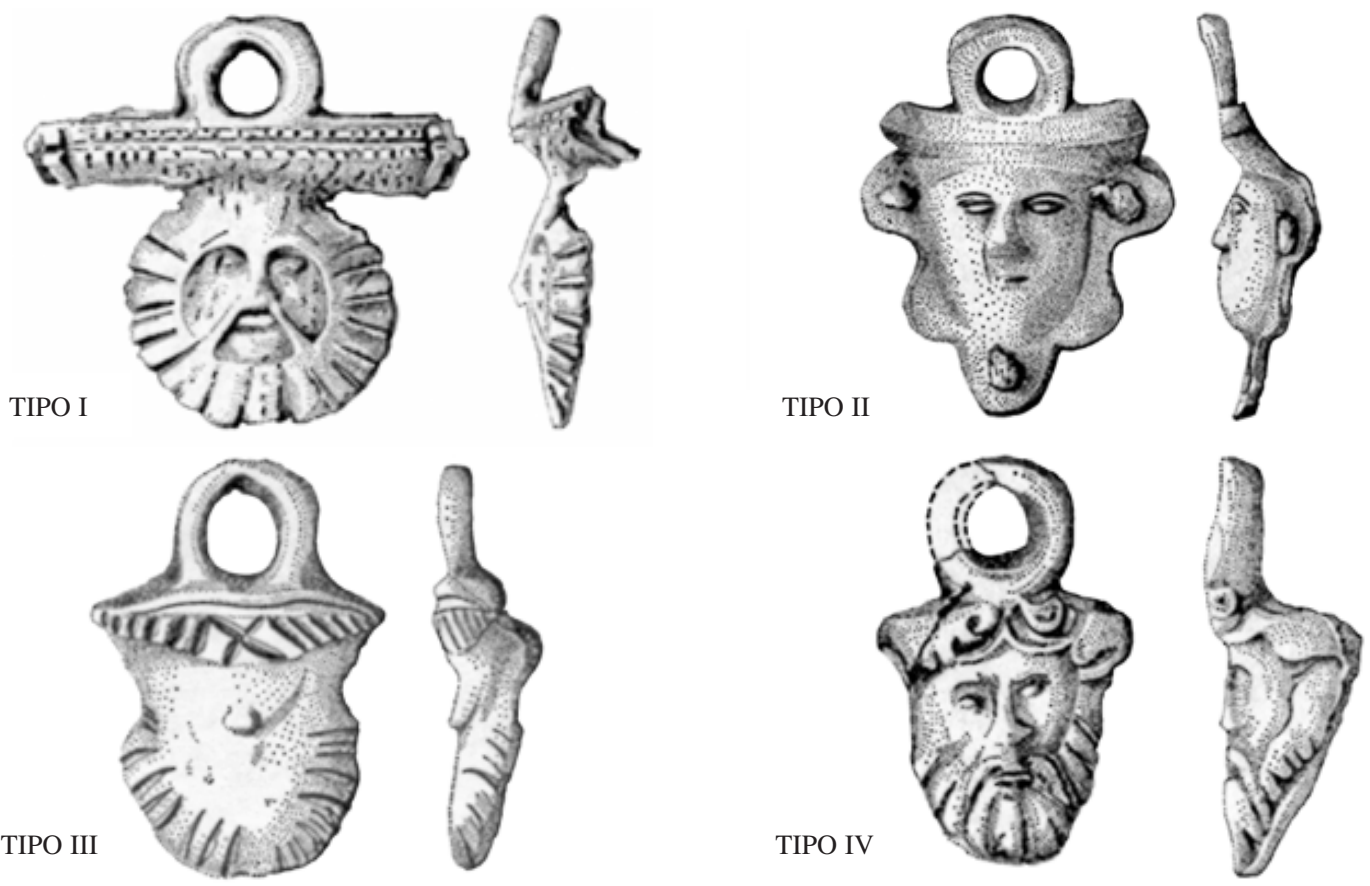

Figura 2. Esquema de los tipos de M. Delgado.

excepcionales (fig. 2). J. Alarcão publica una adquirida por el Museu Nacional Machado de Castro con una procedencia imprecisa, posiblemente de Maceira-Liz (Leiria, Portugal) ${ }^{5}$. La sítula está adornada por dos apliques figurados distintos, uno perteneciente al tipo III y el otro al IV de Delgado -(fig. 3.2)-. Por su parte, G. Marqués dio a conocer un acetre procedente de Torre dos Namorados, (Fundão, Portugal), cuyo único aplique conservado corresponde al tipo I de Delgado -(fig. 3.3)- ${ }^{6}$. Un tercer ejemplar proviene de la mina Fojo das Pombas,

\footnotetext{
$51970,213$.

6 1969, 73-74, lám. IV.3; V.3; XII.3.
}

(Valongo, Portugal) cuyo aplique también pertenece al tipo I de Delgado -(fig. 3.4)- ${ }^{7}$. L. de Alcubierre presenta, además, otra sítula de la misma procedencia, que no ha conservado los apliques, pero que tiene idénticas características formales que la anterior -(fig. 3.5)-.

El quinto ejemplar se encuentra recogido entre las láminas de dibujos del álbum de L. Vives, actualmente en la Hispanic Society of America de Nueva York $^{8}$. El acetre conserva ambos apliques figurados, que son idén-

\footnotetext{
7 L. De Alcubierre e Castro, 1962, 174-175, fig.6.6; 6.11; 6.7.

8 A. García y Bellido (ed.), 1993, 163, lám. 299 y 267. En paradero desconocido. Se desconocen las medidas.
} 


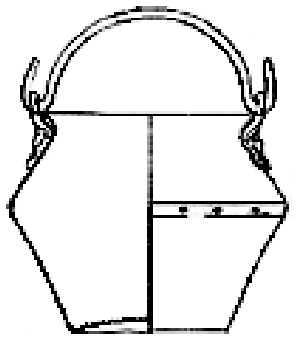

1. Zaragoza

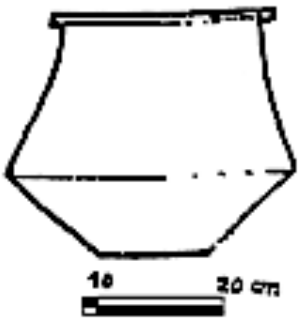

5. Fojo das Pombas, Valongo

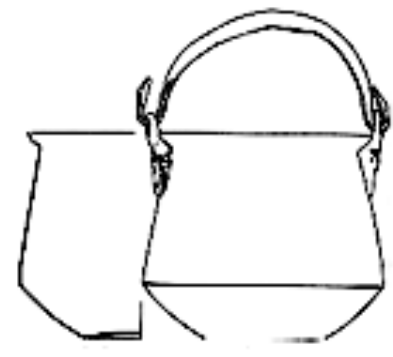

2. Maceira-Liz, Leiria

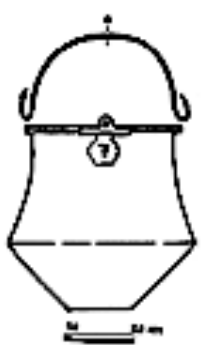

3. Torre dos Namorados, Fundão

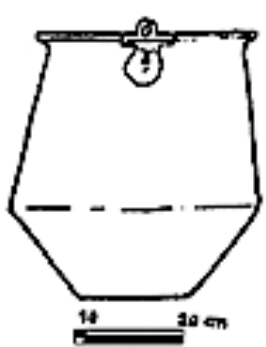

4. Fojo das Pombas, Valongo

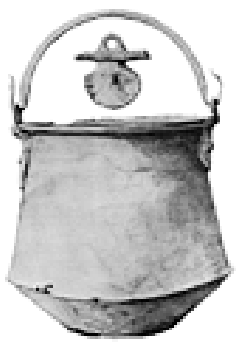

6. Colección Vives

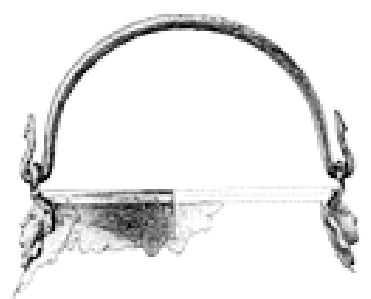

7. La Olmeda, Pedrosa de la Vega, Palencia

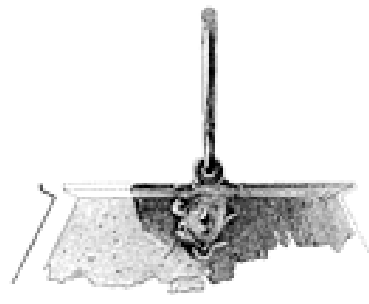

Figura 3. Situlas hispanas con apliques figurados.

ticos entre ellos y catalogables en el tipo I de Delgado -(fig. 3.6)-.

Un sexto recipiente, conservado parcialmente, es el hallado en La Olmeda, Pedrosa de la Vega (Palencia) ${ }^{9}$. El borde y la boca son similares a los anteriormente mencionados, pero falta el cuerpo. Los apliques figurados pertenecen al tipo II de Delgado -(fig. 3.7)-. En la publicación se le presenta relacionado con una base en anillo con tres soportes en forma de garra de felino, al que se uniría por medio de tres remaches conservados. Con gran probabilidad no pertenecerían a la misma pieza, ya que el peso que añadiría la base al cuerpo sería excesivo, y un arreglo más sencillo proporcionaría suficiente estabilidad a la vasija. Las sítulas o acetres no presentan ese tipo de forma de la base, que variaría su funcionalidad, y que es, sin embargo, característico de otro tipo de útiles, como son las authepsae ${ }^{10}$.

Todos los recipientes mencionados muestran características similares. Tienen un cuerpo bitroncocónico, con una carena situada generalmente por debajo de la parte media de la vasija y un borde exvasado. Se fabricaron por martilleado de una sola lámina de bronce, salvo el procedente de Zaragoza, que se llevó a cabo por el trabajo de martilleado de dos láminas y un acabado de

\footnotetext{
9 P. Palol,1970, 222-223, fig.7.36.

${ }^{10}$ T. Tomasevic Buck, 2002, 214-215.
}

trabajo a torno. Los tamaños de los mismos difieren en todos los casos, ya que varían entre $\operatorname{los} 12,8 \mathrm{~cm}$ de la sítula de Maceira-Liz hasta los 28,5 del recipiente procedente del pozo de Torre dos Namorados ${ }^{11}$. Del mismo modo, las bases son todas diferentes. Mientras el fondo exterior del acetre publicado por J. Alarcão es ligeramente cóncavo, la base de la sítula de la Torre dos Namorados presenta la huella de soldadura de un anillo, que serviría de pie en todo su perímetro, y la sítula que conserva un aplique figurado de Fojo das Pombas "tuvo tres pies soldados, de los que no se encontró ninguno" ${ }^{12}$. Estos ejemplares completos y sin arreglos plantean la posibilidad de que la sítula de Caesaraugusta no tenga actualmente la forma original y que el recipiente haya necesitado unos arreglos en sus paredes que hayan variado la forma para permitir la continuación de su uso, o bien se hayan cambiado los apliques y el asa, idea menos probable, a otra vasija reparada y remachada.

La característica común de la forma de las sítulas, y su relación directa con los apliques figurados publicados en la tipología de M. Delgado, salvo en el caso de la procedente de Caesaraugusta, permiten reconocer

\footnotetext{
${ }^{11}$ Fig. 3.2: $12,8 \mathrm{~cm}$; fig.3.3: $28,5 \mathrm{~cm}$; fig.3.4: $27,2 \mathrm{~cm}$; fig.3.5: $19 \mathrm{~cm}$; fig.3.6: desconocido.

12 J. Alarcão,1970, 211; G. Marqués, 1969, 74; L. de Alcu-
} bierre, 1962, 175 

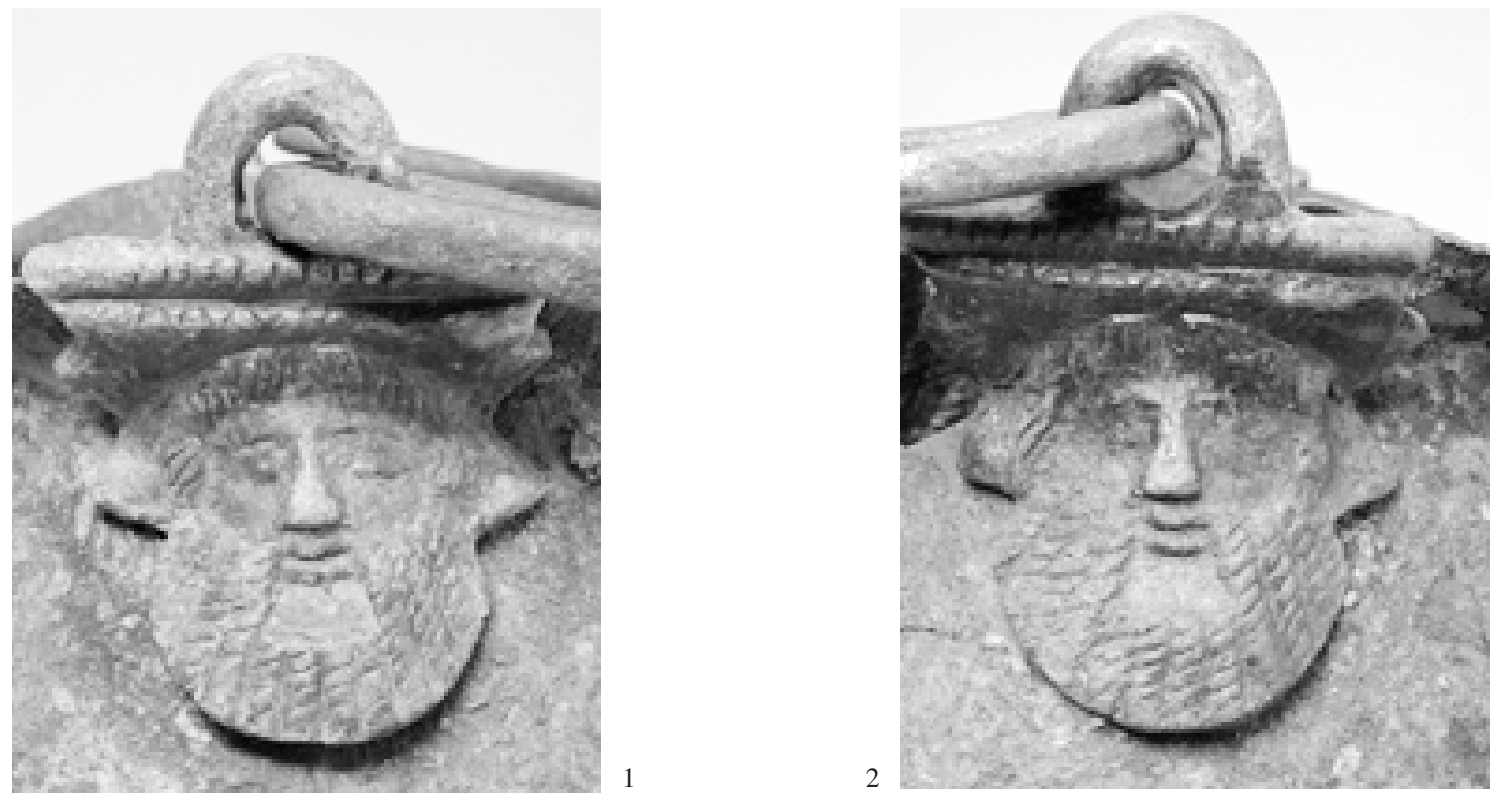

Figura 4. Los apliques de la sítula de Caesaraugusta.

un nuevo tipo incorporable a la tipología de formas de vajilla metálica hispanas. No obstante, el número de hallazgos que lo corroboran, cinco, es ciertamente todavía escaso, y nuevos ejemplares deberán confirmar la propuesta ${ }^{13}$. Del mismo modo, resultaría interesante poder mostrar, en el caso de confirmarse esta hipótesis con el hallazgo de un suficiente número de sítulas, si presentan pequeñas particularidades formales para cada uno de los tipos de apliques figurados propuestos por M. Delgado.

\section{EL ASA}

El asa de bronce se conserva en su posición de uso. El vástago, de sección circular, describe una forma de omega, acabando uno de sus extremos en una cabeza esquematizada de ánade, mientras que el otro sufre un suave estrechamiento del propio vástago. La función a que estaba destinado precisaba de un buen núcleo metálico, por lo que el asa fue fundida en macizo en una sola pieza y, posteriormente, por forja fueron doblados sus extremos.

${ }^{13}$ L. Caballero planteaba ya en 1974 (143) que sobre esta forma "se empleaban los soportes de asas estudiados por Delgado" y, los hallazgos localizados hasta la actualidad parecen igualmente reservar los apliques figurados propuestos por M. Delgado a esta forma.

\section{LOS APLIQUES}

Los dos apliques muestran la misma figura de un rostro masculino barbado con los rasgos faciales claramente diferenciados, pertenecientes al tipo III de Delgado.

El primero mide 7,6 cm de largo y $6,1 \mathrm{~cm}$ de ancho (fig. 4.1 y fig. 5.10). El aplique se encuentra en buen estado de conservación. Los ojos, probablemente almendrados, y la línea de las pestañas han perdido la nitidez del trabajo que se llevaría a cabo tras la fundición. La mirada muestra un rostro distante, ausente. La nariz es triangular y pronunciada. La boca, que permanece cerrada y presenta unos gruesos labios, aparece cuidadosamente incluida entre la abundante barba. Ésta está formada por doce gruesos tirabuzones en los que cada giro del mechón está marcado por incisiones oblicuas. Bajo la boca, y dejando un espacio vacío, se sitúan cuatro de los tirabuzones y a cada lado de éstos se ubica un grupo de otros cuatro, que descienden desde la altura del bigote formando una densa barba redondeada. El cabello abundante y rizado se prolonga por la parte de las orejas hasta la barba. El corto travesaño del aplique se encuentra decorado por dos gruesos baquetones, el superior segmentado por pequeñas incisiones oblicuas, mientras que en el inferior las incisiones dibujan triángulos. Los apéndices laterales triangulares, que suelen estar ocupados por adornos vegetales -hojas de vid pertenecientes a la corona de pámpanos- se encuentran lisos y limpios de decoración, pero bien significados. 
El segundo aplique tiene las mismas dimensiones $7,6 \mathrm{~cm}$ de largo y $6,1 \mathrm{~cm}$ de ancho (fig. 4.2 y fig. 5.11). El aplique fue realizado con el mismo molde que el aplique anterior, por lo que conserva los mismos rasgos faciales, algo perdidos en la parte de los ojos, que se encuentran menos nítidos.

\section{EL TIPO III DE DELGADO}

Las características que diferencian este tipo de apliques de los demás tipos propuestos por M. Delgado son la forma ovalada del aplique decorado con un rostro barbado, así como la atrofia, hasta la práctica inexistencia, del travesaño que se une a la anilla. Este último está funcionalmente representado por una ala trapezoidal saliente, que está adornada con motivos geométricos o vegetales.

Los ejemplares que vienen a continuación presentan estas particularidades, pero además unas características nuevas, que no incluyó M. Delgado en su tipo III, ya que propuso el tipo a partir de un solo ejemplar. De este modo, los apliques de este tipo presentan además unos apéndices triangulares laterales, situados a la altura aproximada de las orejas, que se convierten en la mayoría de los ejemplares en hojas con sus nervios bien diferenciados. La barba del personaje se compone siempre de gruesos tirabuzones, cuyos giros vienen marcados con pequeñas incisiones oblicuas. El bigote se continúa en los gruesos bucles de la barba, dejando cuatro tirabuzones más cortos bajo la boca.

Los ejemplares pertenecientes a esta variante dispersos por la Península Ibérica son los más escasos de entre los correspondientes a la clasificación llevada a cabo por M. Delgado. No existen paralelos fuera de la Península Ibérica, como ocurre con los otros tipos de la misma clasificación, es decir los tipos I, II y IV de esta autora. Se trata, por tanto, de una forma hispana con una amplia difusión peninsular y probablemente con diferencias entre los distintos talleres.

\section{CATÁLOGO}

España- Portugal

Fig. 5.1. Conímbriga, Portugal. Mide $8 \mathrm{~cm}$. de largo. Se encuentra en el Museu Monográfico de Conímbriga, $\mathrm{n}^{\circ}$ inv. 70653. Publicado en A. Moutinho Alarcão (coord.), Coleçoes do Museu Monográfico de Conímbriga, Coimbra 1984, 33, nº 124. Representa el rostro de un hombre maduro con una abundante barba compuesta por gruesos tirabuzones. El bigote se prolonga en dos de los tirabuzones de la barba. Los ojos muestran una mirada inexpresiva, lejana. El corto travesaño es trapezoidal y se adorna con motivos vegetales. A la altura de sus orejas se sitúan unos triángulos, que se corresponden con otros dos ubicados a la altura del travesaño.

Fig. 5.2. Conímbriga, Portugal. Mide $6,2 \mathrm{~cm}$ de largo. Se encuentra en el Museu Monográfico de Conímbriga. Publicado por M. Delgado (1970,24, lám.II.4), es el único ejemplar que constituye el tipo III de la clasificación de esta autora. Salvo una parte de la nariz, ha perdido los rasgos faciales, o bien el grado de esquematización del molde de fundición era tal, que no los contenía. La abundancia de barba se representa por incisiones en todo el borde del espejo.

Fig.5.3. San Sebastiaõ do Freixo, Batalha, Portugal. Publicado por J.M. Bairrao y J. Alarcão, 1969, lám.V.1. Se depositó en el Gabinete de Etnografia de la ciudad de Leiria. Se halló en un pozo durante unas prospecciones llevadas a cabo en el territorio en el año 1963. Representa un hombre maduro con una abundante barba compuesta por gruesos tirabuzones. Los bigotes se prolongan en tirabuzones de la barba, acogiendo cuatro de ellos bajo la boca. En los laterales a la altura de las orejas se sitúan dos triángulos a cada lado, ocupados por hojas. El personaje tiene abundante cabellera que se adentra bajo el travesaño, decorado con motivos geométricos y vegetales.

Fig. 5.4. y fig. 5.5. Santa Menina, Fundão, Portugal. Dos apliques iguales hallados con la anilla de suspensión de la sítula. Publicado por J. Leite Vasconcelos, 1924, 31-32, fig. 2. En la figura de la publicación se observa un rostro masculino redondeado con abundante cabello rizado, una amplia barba también redondeada, compuesta de gruesos tirabuzones y unos bigotes que se prolongan hasta fundirse en los tirabuzones de la barba. Los rasgos del rostro se encuentran claramente diferenciados. El travesaño, por su parte, muestra las características dobles hojas a ambos lados del mismo.

Fig. 5.6. Zafarraya, Granada. Mide $8,4 \mathrm{~cm}$ alto; $6 \mathrm{~cm}$ ancho y $2,9 \mathrm{~cm}$ grosor. Se encuentra en el M.A.N, $\mathrm{n}^{\circ}$ inv. 37.854. Publicada en el catálogo de la exposición de Bronces Romanos, $286 \mathrm{n}^{\circ} 233$. Representa un rostro masculino con abundante barba, recortada de forma redondeada, compuesta por tirabuzones. El rostro está bien definido, los ojos presentan el iris vaciado y los labios se mantienen cerrados. En el travesaño, unas líneas incisas paralelas ocupan el lugar del cabello, mientras que la franja superior está adornada por motivos geométricos y vegetales. Fracturas del metal en la zona de las orejas impiden su descripción.

Fig. 5.7. Mérida. Mide 10,5 cm de alto. Se encuentra en el museo Arqueológico Nacional de Mérida, $n^{\circ}$ inv. 9339. Muestra un estado de conservación que impide describir con precisión algunos detalles. Representa el rostro de un personaje masculino con una barba compuesta por gruesos tirabuzones con pequeñas líneas oblicuas que indican las vueltas de cada mechón. Cuatro tirabuzones, bajo la boca, se encuentran delimitados 


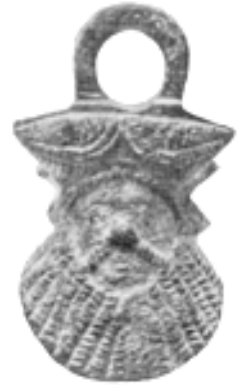

1. Conimbriga

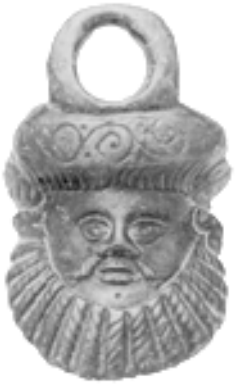

6. Granada

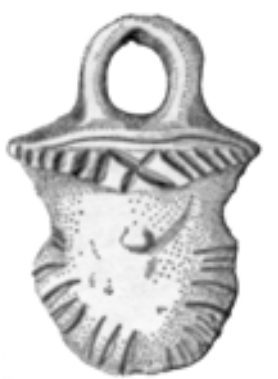

2. Conimbriga

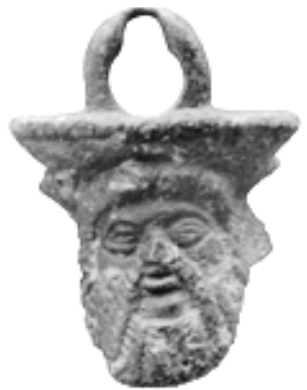

7. Mérida
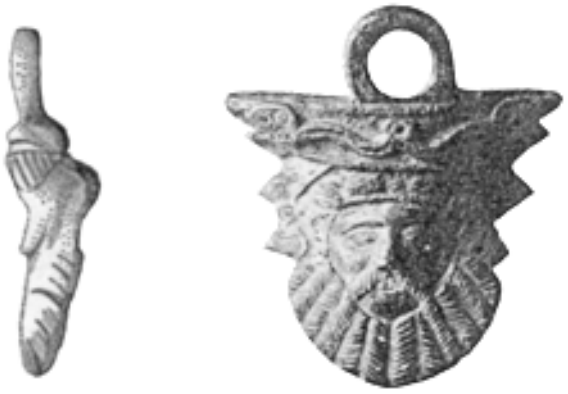

3. San Sebastiaõ do Freixo

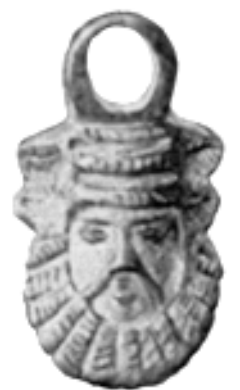

8. Colección Hispanic Society

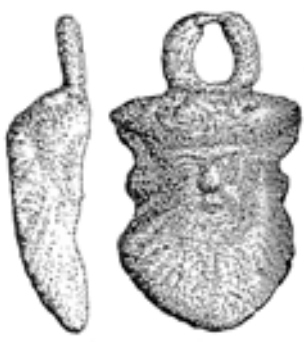

9. Lugo

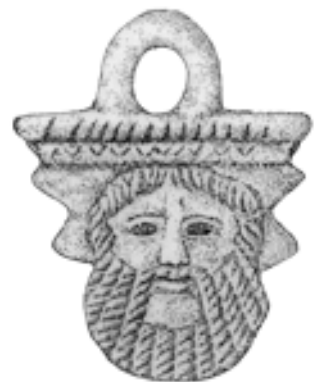

11. Zaragoza

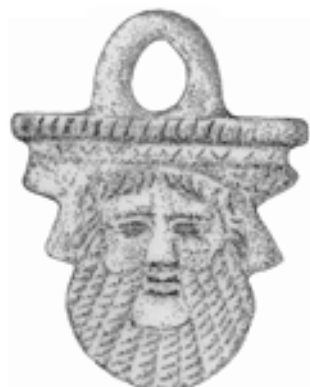

10. Zaragoza

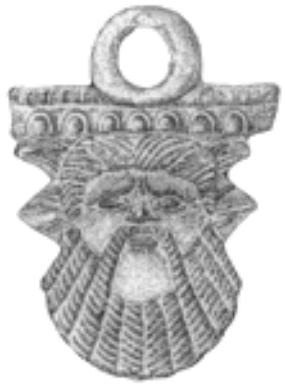

12. Colección Tejerizo

Figura 5. Los apliques tipo III de Delgado.

por los que ascienden para formar el bigote. Los labios se encuentran cerrados y los ojos están bien definidos. La nariz puntiaguda se conserva dañada. Un abundante cabello asciende y ocupa la parte inferior del travesaño. Los laterales a la altura de las orejas están ocupados por dos triángulos a cada lado, sobre los que no se observa ninguna decoración. El corto travesaño del aplique está recorrido por una acanaladura que forma dos baquetones. El ojal ha variado su forma debido al uso, ya que ha ido desgastando el anillo circular original. 
Fig. 5.8. Se encuentra en la Hispanic Society of America, Nueva York, $\mathrm{n}^{\circ}$ inv. R4496. Procedencia desconocida, fue adquirida en Madrid. Publicada por A. García y Bellido (ed), 1993, 199 y 273, lám. 335.7. Mide $6,4 \mathrm{~cm}$ de alto y $3.3 \mathrm{~cm}$ de ancho. Representa un personaje maduro con una barba compuesta por tirabuzones con unas pequeñas incisiones que indican cada giro del bucle. El bigote se prolonga en unos mechones rizados más gruesos, que engloban bajo la boca otros cuatro tirabuzones. A los lados del corto travesaño, con decoración geométrica, emergen dos triángulos a cada lado con los nervios de las hojas bien definidos.

Fig. 5.9. Penadominga, Quiroga, Lugo. Mide $5,9 \mathrm{~cm}$ alto; $5 \mathrm{~cm}$ ancho. Se encuentra en el Museo Provincial de Lugo. Publicada por L.X. Carballo Arceo, 1984. El estado de conservación de la pieza impide una definición clara de todos sus elementos. Representa una figura masculina con abundante barba redondeada, probablemente compuesta por gruesos tirabuzones. Se observan los ojos, los labios cerrados y la nariz del rostro, que se asemeja a los ejemplares de Conímbriga. Conserva unos apéndices laterales triangulares a la altura de las orejas. El travesaño es corto, trapezoidal y parece estar adornado. La anilla de suspensión muestra la huella del desgaste, debido a un uso continuado.

Fig. 5.10. Zaragoza. Mide 7,6 cm de alto y $6,1 \mathrm{~cm}$ de ancho. Se encuentra en el Museo de Zaragoza, $\mathrm{n}^{\circ}$ inv. 01.6.1.(véase la descripción supra)

Fig. 5.11. Zaragoza. Mide 7,6 cm de alto y $6,1 \mathrm{~cm}$ de ancho. Se encuentra en el Museo de Zaragoza, $n^{\circ}$ inv. 01.6.1.(véase la descripción supra)

Fig. 5.12. Pertenece a la Colección Tejerizo, que se encuentra en el Museo de Zaragoza, $\mathrm{n}^{\circ}$ inv. 00.2.10. Su procedencia es desconocida. El aplique mide $9,7 \mathrm{~cm}$ de largo y 7,2 cm anchura el travesaño. La forma del aplique es ovalada. El rostro representado es el de un personaje anciano con el ceño ligeramente fruncido y con una abundante barba compuesta por doce tirabuzones, cuyos rizos se marcan por medio de pequeñas líneas oblicuas incisas. El cuarto tirabuzón asciende por ambos lados hasta el bigote. Los labios, bien marcados, se encuentran cerrados. La nariz es triangular y pronunciada. Los ojos son de forma almendrada y lisos y no tiene diferenciadas las pupilas. Un abundante cabello ocupa el espacio hasta alcanzar el travesaño. Los apéndices triangulares laterales, dos a cada lado, tienen su superficie ocupada por motivos vegetales, unas hojas con sus diferentes partes bien diferenciadas. El corto travesaño tiene como decoración una ranura, que forma dos baquetones. Del superior sólo se observan restos de incisiones paralelas, mientras que el inferior, más ancho, está adornado por una banda de ovas.

Tanto los apliques $n^{\circ} 1,3,4,5,6,7,8,9$ como posiblemente $\operatorname{los} \mathrm{n}^{\circ}, 10,11$ y 12 presentan los apéndices laterales triangulares, algunos de los cuales conservan el motivo vegetal -una hojas- dibujado sobre su superficie. Los

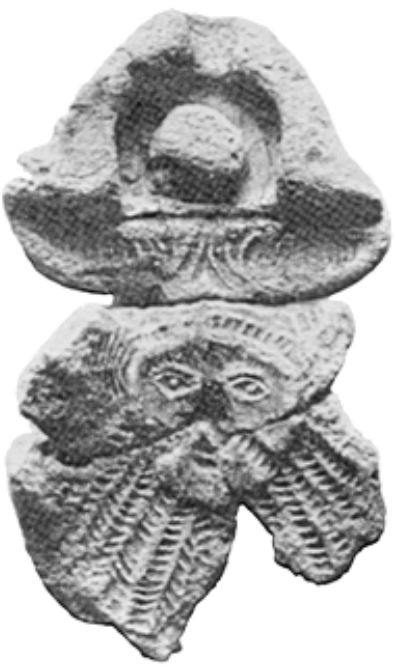

Figura 6. Molde bivalvo procedente de Conímbriga. Foto de A. Moutinho Alarcao y S.da Ponte, 1984, 34, fig. 123.

apliques $n^{\circ} 1,3,4,5,7,8$ y 12 muestran dos hojas a cada lado. Otro elemento común es la forma de la barba compuesta por doce gruesos tirabuzones, como se observa en los apliques $n^{\circ} 3$, probablemente 4 y 5,6,7,8,10,11 y 12 . El cuarto tirabuzón por cada lado asciende formando el bigote, cobijando entre sus dos brazos, y bajo la boca, otros cuatro gruesos rizos. El ejemplar $\mathrm{n}^{\mathrm{o}} 1$ presenta, por otra parte, unas pobladas barbas redondeadas compuestas, igualmente, por gruesos tirabuzones, pero ya ha perdido la característica del prototipo común, que ha guiado la fabricación de los anteriores, como es el albergar entre los bigotes a cuatro gruesos tirabuzones de la barba.

\section{FABRICACIÓN}

La fabricación de los apliques se lleva a cabo por fundición de las piezas en moldes bivalvos, como muestran los distintos fragmentos recogidos en las excavaciones de Conímbriga ${ }^{14}$ (fig. 6). Los apliques eran -en época romana- acoplados y soldados a los bordes de las sítulas, pero existen ejemplares que fueron remachados a la vasija ${ }^{15}$, seguramente formando parte de un arreglo

\footnotetext{
14 A. Moutinho Alarcão, S. Da Ponte, 1984, 34, 33 nº122$123 \mathrm{y}$ fig. 123 .

${ }^{15}$ Como los apliques hallados en Maceira-Liz (J. Alarcao,1970,212,fig.1) y León (T. Mañanes,1983,401,lám.I.14). Los remaches de sujeción del aplique aparecen en posición horizontal en el ejemplar procedente de Maceira-Liz, donde ocupan el lugar en el que se situarían las orejas del personaje y en posición vertical, como en el ejemplar procedente de León, donde los orificios se ubican en la boca de la figura y en el centro del travesaño.
} 
posterior que permitiera una segura fijación y la reutilización del recipiente.

\section{ORIGEN DEL TIPO}

El tipo de aplique en el que figura un hombre maduro con una abundante barba compuesta por gruesos tirabuzones y sobre su cabellera motivos vegetales, como aparece en los tipos I, III y quizá el IV de M. Delgado, tiene relación, como ya mencionara esta autora ${ }^{16}$, "con tipos más antiguos y conocidos por su amplia distribución". J. Aurrecoechea y $\mathrm{M}^{\mathrm{a}}$ del M. Zarzalejos afirmaron que las similitudes encontradas con otros apliques franceses y alemanes "no son paralelos en sentido estricto, sino antecedentes en cuanto a la sintaxis decorativa y conceptual del aplique “ ${ }^{17}$, para añadir más adelantes que "muchos de nuestros ejemplares se hallan inmersos en un lenguaje conceptual existente en otras áreas del Imperio, si bien los resultados finales se presentan aquí con marcada personalidad propia" ${ }^{18}$. Trabajos posteriores comparten la teoría publicada por estos autores ${ }^{19}$.

Siguiendo esta misma línea de interpretación, se puede considerar que el modelo que tratan de copiar o en el que inicialmente se inspiran para llevar a cabo estos apliques es con gran probabilidad el perteneciente a una sítula de época helenística en la que aparece el rostro de un personaje masculino de edad madura con un rostro inexpresivo y una abundante barba rizada, compuesta por gruesos y largos tirabuzones. El cabello es ondulado, adornando su frente con una cinta y, a modo de corona, diversos elementos vegetales (pámpanos y hojas de parra) y corimbos. Unas hojas de vid sobresalen por la parte posterior de la cabeza hasta alcanzar la barba del personaje. Sobre la cabeza se eleva el anillo del aplique en el que se asienta el asa de la sítula. El personaje que aparece es identificado con el dios Dioniso ${ }^{20}$, y su rostro se encuentra en apliques de sítulas, como la encontrada en Meroë (Sudán, hoy en el museo de Munich), considerada el prototipo más antiguo; o la hallada en la isla de Delos (Grecia); además de la aparecidas en Ostia, en Pompeya, en Celeia (Eslovenia, hoy en el Kunsthistoriches Museum de Viena) y en Ani (Armenia), junto a otros ejemplares también enumerados por I. Jenkins ${ }^{21}$ de procedencia desconocida, pero conservados en los muse-

\footnotetext{
$161970,18-19$.

$171990,287$.

${ }^{18} 1990,291$.

${ }^{19}$ R. Castelo, P. Gómez, A. Torrecilla, R. Arribas, I. Panizo, 1995,130-131.

${ }^{20}$ I. Jenkins, 1994,273-299.

${ }^{21}$ I. Jenkins, 1994,277,279.
}

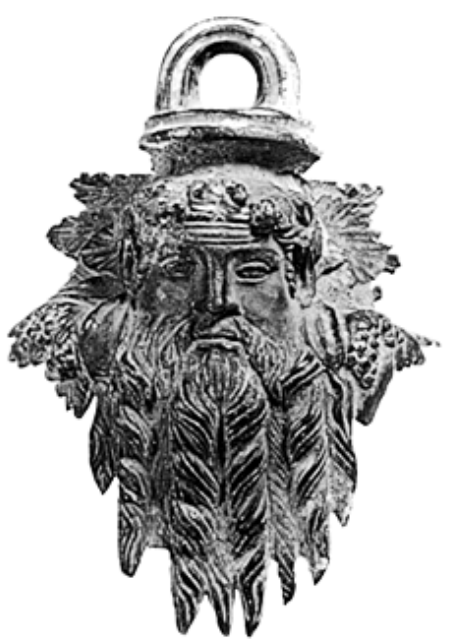

Figura 7. Aplique con máscara procedente del Musée du Petit Palais, París.

os de Nueva York, British Museum, Colchester Castle Museum y en el museo du Petit Palais de Paris (fig. 7). La fecha del grupo más antiguo, según I. Jenkins ${ }^{22}$, se sitúa en el siglo II a.e., y el tipo sigue produciéndose durante el siglo I d.e. (como dan testimonio los ejemplares procedentes de Atenas, Roma y Ancona). De este prototipo existen también ejemplares hispanos en cerámica, como el expuesto en el Museo Arqueológico de Alicante, procedente del yacimiento de Lucentum-Tossal de Manises (Alicante), y el ejemplar originario de las excavaciones de San Antón, Cartagena ${ }^{23}$.

Los ejemplares del tipo III de Delgado que se presentan en este trabajo muestran, mejor que ningún otro tipo de aplique figurado hispano, la vinculación con los modelos evolucionados de los tipos helenísticos. Tanto las hojas que adornan la corona de Dioniso, que aparecen únicamente sobre el tipo III de Delgado, como la barba de gruesos y largos tirabuzones, la abundante cabellera y la mirada inexpresiva propia del dios son rasgos y características tomadas de un prototipo que, como hemos visto tuvo una amplia difusión. No se trataría, por tanto, de ninguna cabeza de sátiro, ni de Aqueloo ${ }^{24}$, sino de Dioniso, una divinidad bien conocida durante la Antigüedad Clásica y extensamente representada en otros soportes como mosaicos, estatuaria, sarcófagos, cerámica, etc.

\footnotetext{
${ }^{22}$ I. Jenkins, 1994,284.

${ }^{23}$ MARQ, sala de época romana, vitrina 6. La pieza está fechada en el s. I d.e. La terracota de Cartagena está fechada en el s. I a.e.

${ }^{24}$ L. Caballero, 1974, 142. A. García y Bellido (Ed), 1993, $273,7(484)$ y nota 41.
} 


\section{CRONOLOGÍA}

Ninguno de los ejemplares que hasta hoy forman parte del tipo III de Delgado ofrece una datación estratigráfica. En el interior de la sítula de Caesaraugusta apareció un plato de una forma 15/17 de T.S.H.Intermedia, cuya cronología se sitúa en el siglo III d.e., y un pequeño fragmento de cerámica imperial con pintura a bandas, cuya producción se fecha en los siglos I y II d.e. ${ }^{25}$. Los materiales hallados en el interior de la cloaca, junto a la sítula, fechan el final de uso de dicha estructura a mediados del siglo III d.e. ${ }^{26}$.

Los primeros trabajos portugueses atribuían una fecha "con reservas", proporcionada por los hallazgos en la mina Do Fojo das Pombas, Valongo (Portugal), en el siglo II d.e. Este conjunto permitió a M. Delgado proponer la misma cronología "no segura" a su tipología publicada sobre la base del conjunto de materiales recuperados en las excavaciones de Conímbriga ${ }^{27}$. M. Rosas situó el periodo de uso del ejemplar hallado en Sant Josep, tipo I de Delgado, en la segunda mitad del siglo III y en el IV ${ }^{28}$. J. Aurrecoechea mencionó, en su trabajo sobre la vajilla de los museos de Ciudad Real, que los tipos hispanos I y IV de Delgado tienen una cronología situada entre los siglos II-III d.e., mientras que el tipo II de la misma autora tendría un periodo de uso entre los siglos III y V d.e. ${ }^{29}$.

El hecho de que el tipo III de Delgado conserve un mayor número de características y rasgos de los prototipos originales permite pensar que fuera un tipo hispano anterior al tipo I, es decir más antiguo, y en el que el interés por copiar elementos de los prototipos romanos era todavía importante. Un hecho que en el tipo I no aparece reflejado, ya que se observa una esquematización de los rasgos recogidos en el tipo III. Por tanto, y a la espera de los datos que proporcionen nuevos hallazgos, pero a la vez teniendo en cuenta que el lugar de hallazgo de la sítula de Caesaraugusta fue destruido a mediados del siglo III, se puede proponer una cronología para el tipo III de Delgado -con la precaución que se debe tener cuando sólo se cuenta con un hallazgo-, que abarque el siglo II y la primera mitad del III d.e., siendo el resto de tipos de Delgado ligeramente posteriores.

\footnotetext{
25 Agradezco los datos proporcionados por las Dras. Pilar Sáenz Preciado y Carmen Aguarod Otal relativos a los fragmentos cerámicos mencionados.

${ }^{26}$ Blanca del Real, en el informe que nos ha proporcionado, menciona fragmentos de TSH lisa y decorada, así como fragmentos de TSHt, de cerámica de cocina africana, de cerámica engobada, de comunes de cocina y de almacenaje, así como restos de pintura mural romana y materiales de construcción.

${ }^{27}$ L. de Alcubierre, 1962,168; M. Delgado,1970,19.

${ }^{28} 1980,205$, fig.2.17

291991,248 .
}

\section{FUNCIÓN}

Los acetres o sítulas que han llegado hasta nuestros días muestran huellas de un gran uso. Fueron reparadas una y otra vez. Cuando se deterioraban, se cambiaba el asa, se reponían los apliques -incluso remachando algunos de ellos- y se llevaban a cabo reparaciones en la lámina que formaba el recipiente, tanto en las paredes, como en el borde. Todo ello evidencia un uso continuo y frecuente.

Las sítulas con apliques decorados pertenecen al ajuar de bebida del servicio de mesa ${ }^{30}$ y debían contener y transportar agua y vino ${ }^{31}$, además de las distintas mezclas de ambos líquidos. Seguramente, esta misma función prestarían las sítulas adornadas por apliques del tipo III de Delgado, siguiendo el uso de los recipientes helenísticos con apliques que recogen el rostro del dios Baco - el Dioniso griego -, que les sirvieron de modelo. No sería extraño que al tener la misma función, se imiten también los elementos de adorno.

El hallazgo de las sítulas procedentes de Torre dos Namorados en el interior de un pozo de agua, podría vincular su uso a simples baldes o cubos que cayeron al fondo y se abandonaron ${ }^{32}$. Sin embargo, para rebatir esta conjetura no hay que olvidar la reflexión expuesta por M. Pernot ${ }^{33}$ relativa a que algunos de estos sistemas de apliques son incapaces de resistir la decena de kilos que debería soportar una sítula llena de líquido. Si a esto añadimos que cuando se quiere extraer agua de un pozo se necesita, además, un recipiente que aguante cierto peso, golpes y tirones, parece claro que la sítula no sería el recipiente más adecuado para dicha función.

\section{ADDENDUM}

En el Museo Arqueológico Municipal de Alcoi se encuentra recogido un aplique que puede sumarse a la lista del tipo III de Delgado. El aplique - $n^{\circ}$ inv. 1.179procede de Els Plans (Alcoi), mide 6,85 $\mathrm{cm}$ de largo y 5

\footnotetext{
30 J. Kunow, 1983,79,83; R. Petrovszky,1994, 677.

31 A. Radnóti, 1938,112; M.Bolla, C.Boube, J-P.Guillaumet,1991,22; K. Szabó,1995, 80; I.Jenkins, 1994, 288. También se han atribuido otros usos a determinadas sítulas, como contenedor de la sangre de animales durantes ritos con sacrificios ( $\mathrm{J}$. Pollini, 2002,35) o una utilización secundaria de estos recipientes como urna cineraria (M.Bolla, C.Boube, J-P.Guillaumet, 1991,19), además de su presencia en el ajuar para el aseo y la limpieza corporal. Una sítula aparece representada en una escena de aseo de un mosaico -fechado a fines del siglo IV o comienzos del siglo V- de las termas privadas de Sidi Ghrib en Cartago. En la escena la dueña, rodeada de enseres de limpieza, es ayudada por dos sirvientas (B.Baratte, 1997,130-131,fig.22).

${ }^{32}$ G. Marques, 1969,80.

${ }^{33}$ M.Bolla, C. Boube, J-P Guillaumet, 1991,21.
} 
$\mathrm{cm}$ de ancho. Se encuentra publicado en J. E. Aura, J. M. Segura (Coords.), Catálogo del Museu Arqueológic Municipal Camil Visedo Moltó, Alcoi. Alcoi 2000.

\section{BIBLIOGRAFÍA}

ALARCÃO J., 1970, "Um caldeiro romano de bronze", Ethnos VII, 211-213.

ALBURQUERQUE E CASTRO L., 1962, "Achados romanos na Mina do Fojo das Pombas (Valongo)", Estudos, Notas e Trabalhos do Serviço de Fomento Mineiro, 15 3-4, 431-448.

ALCUBIERRE E CASTRO L. 1962, "Hallazgos romanos en el mina "Do Fojo das Pombas", Valongo (Portugal), AEspA. XXXV, 166-176.

AURRECOECHEA J., ZARZALEJOS M $^{\text {a }}$ M., 1990, "Apliques de sítula de la Oretania, algunas matizaciones a la tipología de Delgado", AEspA, 63, 284-292.

AURRECOECHEA J., 1991, "Vajilla metálica de época romana en los museos de Ciudad Real, Jaén y Linares", Espacio, Tiempo y Forma, Serie II, $\mathrm{H}^{\mathrm{a}}$ Antigua, t.IV, 223-254.

BAIRRÃO J.M., ALARCÃO J., 1969, "Excavaçoes em S. Sebastiao do Freixo (Concelho da Batalha)", Conimbriga VIII, 1-12, lám. I a V.

BARATTE F., 1997, "La vaisselle d'argent dans l'Afrique romaine et Byzantine", AnTard 5, 111-132.

BOLLA M., BOUBE CH., GUILLAUMET J-P, 1991, "Les situles", en M. Feugère y $\mathrm{Cl}$. Rolley, La vaisselle tardo-républicaine en bronze, Dijon, 7-22.

BRONCES ROMANOS EN ESPAÑA, 1990, Catálogo de la exposición mayo-julio 1990, Madrid.

CABALLERO ZOREDA L., 1974, "La necrópolis tardorromana de Fuentespreadas (Zamora). Un asentamiento en el Valle del Duero", E.A.E, 80.

CARBALLO ARCEO, L. X., 1984, "Duos novos soportes de asas de situlas de epoca romana", Boletin do Museo Provinciale de Lugo, tomo II, 235-239.

CASTELO R., GÓMEZ RAMOS P., TORRECILLA A., ARRIBAS R., PANIZO I., 1995, "Apliques de asa de sítula con decoración antropomorfa procedentes de la villa romana de El Saucedo (Talavera La Nueva, Toledo)", CuPAUAM, 22, 125-164.

DELGADO M., 1970, "Elementos de sítulas de bronze de Conimbriga", Conimbriga IX, 15-40.

FUENTES A., 1990, "Los bronces bajoimperiales en Hispania", 117-135 en Los Bronces romanos en España, catálogo de la exposición de mayo-julio 1990, Madrid.
GARCÍA Y BELLIDO A. (edit), 1993, Álbum de dibujos de la colección de bronces antiguos de Antonio Vives Escudero, Anejos de AEspA. XIII, Madrid.

JENKINS I., 1994, "The Mask of Dionysos/Pan-OsirisApis", Jahrbuch des Deutschen Archäologischen Instituts, 109, 273-299.

KUNOW J., 1983, Der römische Import in der Germania libera bis zu den Markomannenkriegen, Studien zu Bronze- und Glasgefässen. Neumünster.

LEITE VASCONCELOS J., 1924, "Figuras de bronze antigas do Museu Etnológico Português", O Arqueologo Português XXVI, 29-36, fig. 2.

MAÑANES T., 1983, "Bronces romanos en la Provincia de León”, Homenaje al Prof. Martin Almagro Basch, III, Madrid, 399-410.

MARQUÉS G., 1969, "O poço da estaçao romana de Torre dos Namorados (Fundão)", Conimbriga VIII, 65-83 y láms. I a XII.

MOUTINHO ALARCAO A., DA PONTE S., 1984, Catálogo de las colecçoes do Museu Monográfico de Conimbriga, Coimbra.

PALOL P. de, 1970, "Necrópolis hispanorromanas del siglo IV en el Valle del Duero. III. Los vasos y recipientes de bronce". Boletín del Seminario de Estudios de Arte y Arqueología XXXVI, 222-223.

PETROVSZKY R., 1994, "Die Bronzegefässe", 677, 663-700, en G. Hellenkemper y otros (ed), Das Wrack, der antike Schiffsfund von Mahdia, Köln.

POLLINI J., 2002, Gallo-Roman Bronzes and the Process of Romanization, the Cobannus Hoard, Leiden-Boston.

RADNÓTI A., 1938, Die römischen Bronzegefässe von Pannonien,

ROSAS ARTOLA M., 1980, "El mobiliari metal-lic del poblat ibero-romá de Sant Josep (La Vall D’Uixo, Castelló)", Cuadernos de Prehistoria y Arqueología Castellonense 7.

TOMASEVIC BUCK, T., 2002, "Authepsae, auch ein Instrument der ärztlichen Versorgung?", Acta of the $13^{\text {th }}$ International Bronze Congress, From the Parts to the Whole: Cambridge, Massachussets, 1996, vol. 2, 213-232.

SZABÓ K., 1992, "Situles de Pannonie", Acta of the $12^{\text {th }}$ International Congress on Ancient Bronzes, Nijmegen, 77-85.

Recibido el 11-11-05 Aceptado el 15-03-06 